\title{
Mating behavior induces selective expression of Fos protein within the chemosensory pathways of the male Syrian hamster brain
}

\author{
Sara S. Kollack and Sarah W. Newman \\ Department of Anatomy and Cell Biology, University of Michigan. Ann Arhor, MI 48109-0616 (USA)
}

(Received 3 March 1992; Revised version received 20 May 1992: Accepted 28 May 1992)

Key words: Mating behavior; Proto-oncogene; Fos; Immunocytochemistry; Limbic system: BNST; MPOA; Amygdala

\begin{abstract}
The effect of mating behavior on the expression of Fos protein was analyzed within the chemosensory pathways of the male Syrian hamster brain. Following a single mating test, the number of Fos-immunoreactive (Fos-ir) neurons increased within the amygdala, bed nucleus of the stria terminalis and medial preoptic area. The mating-induced pattern of Fos expression within these brain regions shows a strong correlation with the sites of lesions that eliminate or alter mating behavior. In addition. Fos expression was increased within the paraventricular nucleus of the hypothalamus. These results provide the first demonstration of a dynamic and selective pattern of neuronal activity within specific nuclei known to be essential for mating behavior in the male Syrian hamster.
\end{abstract}

Mating behavior in the male Syrian hamster (Mesocricetus auratus) is dependent on chemosensory cues and gonadal steroids. Lesions placed within the vomeronasal and olfactory pathways that block the processing of sexually-relevant odors eliminate mating (reviewed in ref. 20). Likewise, a reduction in gonadal steroids following castration prevents mating, although the deficits develop gradually over 12 weeks and are reversible (reviewed in ref. 20). Neurons that accumulate gonadal steroids are abundant within specific chemosensory nuclei: the medial nucleus of the amygdala (Me), the bed nucleus of the stria terminalis (BNST), and the medial preoptic area (MPOA) [3, 26]. Thus, these regions may integrate essential chemosensory and hormonal signals.

We have used Fos immunocytochemistry to identify specific subsets of neurons within the Me, BNST, and MPOA that are active during, and therefore important for, mating behavior. Fos protein and mRNA, the cellular products of the c-fos gene, are used as markers of neuronal activation $[6,16,21]$. Indeed, recent work indicates that sexual behavior increases Fos expression within the central nervous system of male rats [1, 7, 19]. However, these studies did not address in detail the role of amygdaloid subnuclei in the processing of chemosensory information nor did they quantify the level of Fos

Correspondence: S.W. Newman. Department of Anatomy and Cell Biology. 5710 Medical Science II Bldg., University of Michigan, Ann Arbor. MI 48109-0616, USA. induction. Thus, the aim of this study was to quantify the mating-induced expression of Fos protein within specific subnuclei along the chemosensory pathways of the male Syrian hamster brain.

Adult male and female Syrian hamsters (110-150 g; Charles River Laboratories), were housed by sex in groups of 4 to 6 per cage in one room under a $14: 10 \mathrm{~h}$ light--dark cycle with food and water provided ad libitum. Twelve sexually naive adult male hamsters were divided into three groups: (1) unmated $(n=6)$, (2) mated and perfused immediately after testing $(n=3)$, and (3) mated and perfused $1 \mathrm{~h}$ after the end of testing $(n=3)$. Mating tests lasted $55 \mathrm{~min}$ and were conducted within the first $4 \mathrm{~h}$ of the dark cycle under dim light. Each mated hamster was allowed to copulate with a series of three females during a single mating test. Sexual receptivity was induced in ovariectomized female hamsters by injections of $10 \mu \mathrm{g}$ estradiol 48 and $24 \mathrm{~h}$ prior to, and $500 \mu \mathrm{g}$ progesterone $4-6 \mathrm{~h}$ prior to, testing. Unmated hamsters remained in their home cages.

All hamsters were deeply anesthetized with sodium pentobarbital and perfused through the aorta with $50 \mathrm{ml}$ of $0.1 \mathrm{M}$ sodium phosphate buffered saline containing $0.1 \%$ sodium nitrite, followed by $200 \mathrm{ml}$ of $4 \%$ paraformaldehyde in $0.1 \mathrm{M}$ sodium phosphate buffer ( $\mathrm{NaPB}$ ). Brains were postfixed for $1 \mathrm{~h}$ at room temperature and then cryoprotected in a $20 \%$ sucrose solution in $0.1 \mathrm{M}$ $\mathrm{NaPB}$ at $4^{\circ} \mathrm{C}$. Coronal sections $(40 \mu \mathrm{m})$ were cut on a freezing microtome and processed for Fos immunocyto- 
chemistry in: (1) rabbit anti-c-Fos antiserum (diluted 1:1000 in potassium phosphate buffered saline (KPBS) with $0.3 \%$ Triton; Ab-2, Lots 10910102 and 109300; Oncogene Sciences) for $48 \mathrm{~h}$ at $4^{\circ} \mathrm{C}$, (2) biotinylated donkey anti-rabbit IgG (diluted 1:100 in KPBS with $0.3 \%$ Triton; Jackson ImmunoResearch Labs) for $1 \mathrm{~h}$, (3) avidin-biotin complex (Vectastain ABC Kit) for $1 \mathrm{~h}$, and (4) $0.0125 \%$ diaminobenzidine (DAB) solution containing $0.06 \%$ hydrogen peroxide and $0.015 \%$ nickel chloride in KPBS for $10 \mathrm{~min}$. All incubations were carried out at room temperature unless otherwise noted. Some sections were counterstained with Cresyl violet.

The rabbit anti-c-Fos antiserum (Ab-2) is directed against the amino terminus (amino acids 417) of the human Fos protein [24] and reacts with c-Fos, Fos-related antigens, and the Fos-Jun heterodimer (Oncogene Sciences). Fos staining was localized to the nucleus of neurons and was eliminated by omission of primary antibody or by preincubation of Fos antiserum with $0.6 \mu \mathrm{M}$ Fos peptide (peptide-2; Oncogene Sciences).

The pattern of Fos-immunoreactive nuclei (Fos-ir neurons) was plotted on tracings of Me, BNST. and MPOA drawn from adjacent Cresyl violet-stained sections (Figs. 1B and 3B). In addition, the mean number of Fos-ir neurons was determined for 20 different subnuclei by averaging cell counts per subnucleus per group (Figs. $1 \mathrm{~A}$ and $3 \mathrm{~A}$ ). For each subnucleus in each brain, the cell count represents an average of 2 to 3 samples taken from different sections. The area analyzed in each sample was $0.21 \mathrm{~mm}^{2}$. In general, the pattern of Fos induction was similar in the brains of hamsters in the two mated groups; thus, data from these two groups were combined for statistical analysis. The levels of Fos expression among subnuclei in the non-mated group (basal expression), and between non-mated and mated groups (mating-induced expression), was assessed using a one-factor and two-factor repeated measures ANOVA, respectively. Post-hoc comparisons were based on the Scheffe method using a 0.05 level of significance. All statistical tests were run with Statview software.

Basal Fos expression differed among the subnuclei $(P<0.001)$, ranging from as low as 6 Fos-ir neurons per $0.21 \mathrm{~mm}^{2}$ within the amygdalohippocampal area (AHA) to as high as $67 \mathrm{Fos}$-ir neurons per $0.21 \mathrm{~mm}^{2}$ in the anterior cortical nucleus of the amygdala (ACo) (Figs. 1A and $3 \mathrm{~A}$ ). The mating-induced expression of Fos protein showed specificity in the distribution of Fos-ir neurons within and among subnuclei $(P<0.005)$ and in the number of neurons actually immunostained for Fos $(P<0.001)$ (Fig. 1A and 3A). The magnitude of Fos induction in each subnucleus is illustrated as the fold-increase in Fos-ir neurons and was determined by dividing the mean number of Fos-ir neurons in the mated group by that of the non-mated group and subtracting 1.0.

Within the amygdala, 7 of the 9 regions studied showed significant increases in Fos expression following mating behavior (Fig. 1A). Large increases in the number of Fos-ir neurons were detected within the caudal part of the posterodorsal subdivision of Me (cMePD), confined largely to the dorsal and lateral parts of this region (Figs. 1A, Biii and 2A,B), and within the AHA (Fig. 1A). Smaller increases were detected within the anterodorsal (MeAD), anteroventral (MeAV) and the rostral part of the posterodorsal ( $\mathrm{rMePD}$ ) subdivisions of $\mathrm{Me}$, the posteromedial cortical nucleus (PMCO) and the posterolateral cortical nucleus (PLCo) (Fig. IA,Bi-iii). No change in Fos expression was detected in the posteroventral subdivision of Me (MePV) or the ACo (Fig. (A, Bi,ii).

A similarly diverse pattern of Fos induction occurred in the BNST and MPOA. Small increases in the number of Fos-ir neurons were observed within the posterointermediate subdivision of BNST (BNSTpi) and throughout the rostrocaudal extent of the medial preoptic nucleus (MPN) (Fig. 3A,Bi iii). More dramatic increases were observed within the magnocellulat MPN (MPNmag) (Figs. 2C.D and 3A,Biii) and the posteromedial subdivision of the BNST (BNSTpm) (Fig. 3A.Bii iii). Fos expression was not significantly increased in the anteromedial subdivision of BNST (BNSTam) (Fig. 3A,Bi).

For comparison with observations in the rat $[1,7,19]$. Fos expression was analyzed within the caudal piriform cortex (PIR) (Fig. 1A). the nucleus accumbens (NAcc), and the paraventricular nucleus of the hypothalamus (PVN) (Fig. 3A). In addition, Fos expression was analyzed within the ventral lateral septum (LSv) for comparison with other gonadal steroid-accumulating regions in the chemosensory pathways $[3,26]$. The number of Fos-ir neurons increased only along the mid-ventral border of the PVN (Figs. 2E.F and 3A). No significant increases were identified in the other subnuclei, although there were more Fos-ir neurons in these areas in mated hamsters than in nonmated hamsters.

Mating behavior resulted in a selective expression of Fos protein in specific nuclei of the chemosensory pathways. The induction of Fos protein in Me. PMCo. and AHA. but not in ACO or PIR, suggests greater activation of the vomeronasal pathway than the olfactory pathway, where only the PLCo nucleus was activated. Further, Fos protein was expressed selectively within specific subdivisions of activated nuclei. For example. Fos immunostaining identified two distinct clusters of neurons in cMePD that are nol distinguishable from surrounding cells in cresyl violet-stained material, nor have 
A

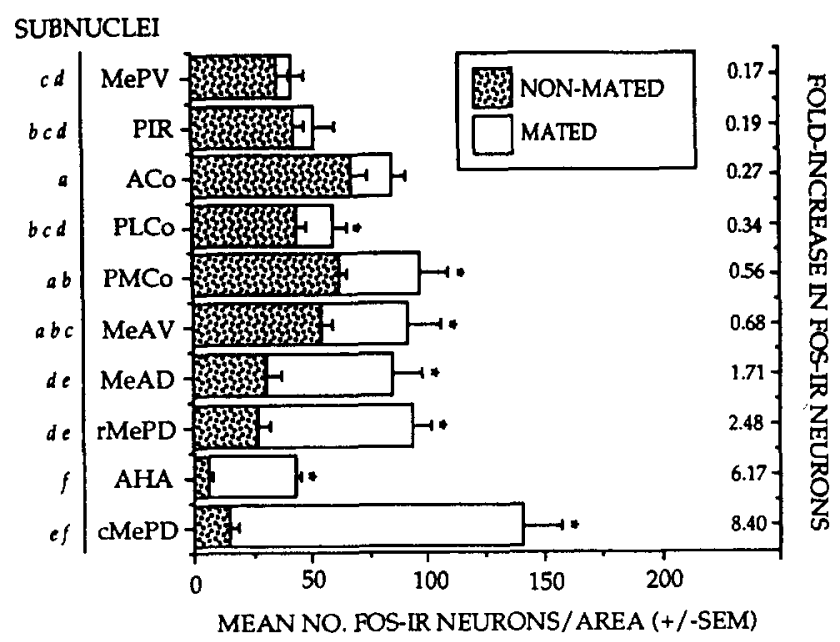

B
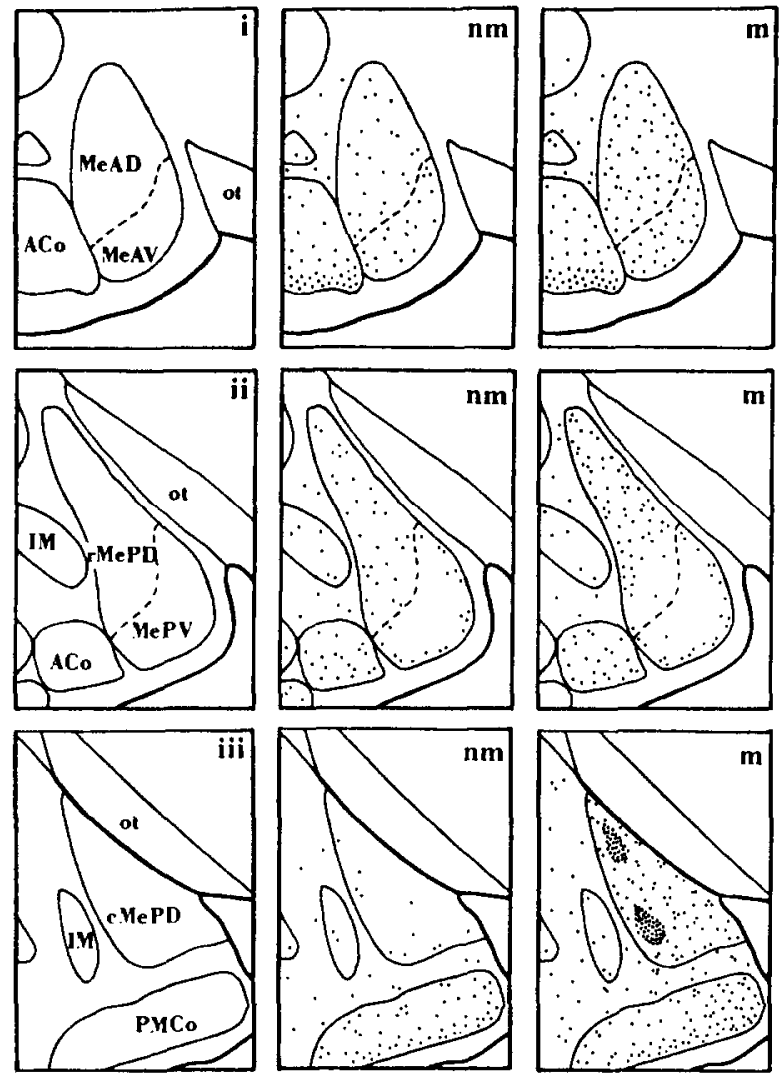

they been selectively identified in previous studies of neurotransmitters, gonadal steroid receptors, or connections of this region. In addition, among these activated regions the magnitude of Fos induction varied. The small and large increases observed in Fos expression may indicate differential levels of neuronal activation.

The pattern of Fos induction within Me, BNST and MPOA shows a strong correlation with the sites of lesions that eliminate or alter mating behavior. Lesions of the anterior Me eliminate mating behavior, while lesions $\leftarrow$

Fig. 1. A: mean number of Fos-ir neurons per subnucleus in the amygdala and piriform cortex of non-mated and mated groups. The fold-increase in Fos-ir neurons indicates the mating-induced increase in Fos-ir neurons above basal levels. An asterisk identifies those subnuclei that contained significantly more Fos-ir neurons following mating. The letters to the left of each subnucleus identify differences between subnuclei in non-mated animals. Subnuclei that share a common letter do not differ significantly. B: 9-panel schematic illustrates the distribution of Fos-ir neurons between non-mated $(\mathrm{nm})$ and mated $(\mathrm{m})$ groups at three different anatomical levels through $\mathrm{Me}$. ACo, anterior cortical nucleus; AHA, amygdalohippocampal area: IM, intercalated mass: Me, medial nucleus; MeAD, anterodorsal Me; MeAV, anteroventral Me; MePV. posteroventral Me: rMePD, rostral part of posterodorsal Me; $\mathrm{cMePD}$. caudal part of posterodorsal Me; PIR, piriform cortex (caudal); PMCo. posteromedial cortical nucleus; PLCo, posterolateral cortical nucleus; ot, optic tract.

of the posterior Me and PMCo alter the temporal sequencing of the behavior [9-11]. Lesions within BNST can selectively reduce chemoinvestigation, whereas lesions of MPOA eliminate copulation [17]. The area common to lesions that reduced chemoinvestigation was centered along the border between BNSTpm and BNSTpi; lesions effective in eliminating copulation were centered in MPNmag. Interestingly, the largest increases in Fos expression occurred within BNSTpm and MPNmag.

Gonadal steroid-accumulating neurons are abundant within cMePD, AHA, BNSTpm and cMPN [26], suggesting the possibility that steroids may influence c-fos expression in these areas during mating behavior. Recent studies have reported that gonadal steroids can induce the expression of Fos antigens within brain $[2,8]$. In addition. an estrogen responsive element has been identified within the promoter region of the c-fos gene [25]. It will be necessary to determine if the chemosensory-induced rise in plasma testosterone levels observed in mated hamsters [13] is important for the pattern of Fos induction observed in this study. It is of interest to note that the LSv did not show a similar change in the number of Fos-ir neurons following mating behavior, even though this region contains a large population of gonadal steroid-accumulating neurons.

In general. our results correlate well with the pattern of Fos induction observed following sexual behavior in the male rat $[1,7,19]$. However, we were unable to detect significant increases in Fos expression in PIR, NAcc, and central tegmental field (not shown). These differences may result from a species-specific pattern of neuronal activation during mating behavior. or from a differential capacity for Fos detection by distinct Fos antisera.

The pattern of activation within the PVN may identify a specific subset of neurons that are important for coordinating the function of the autonomic nervous system 

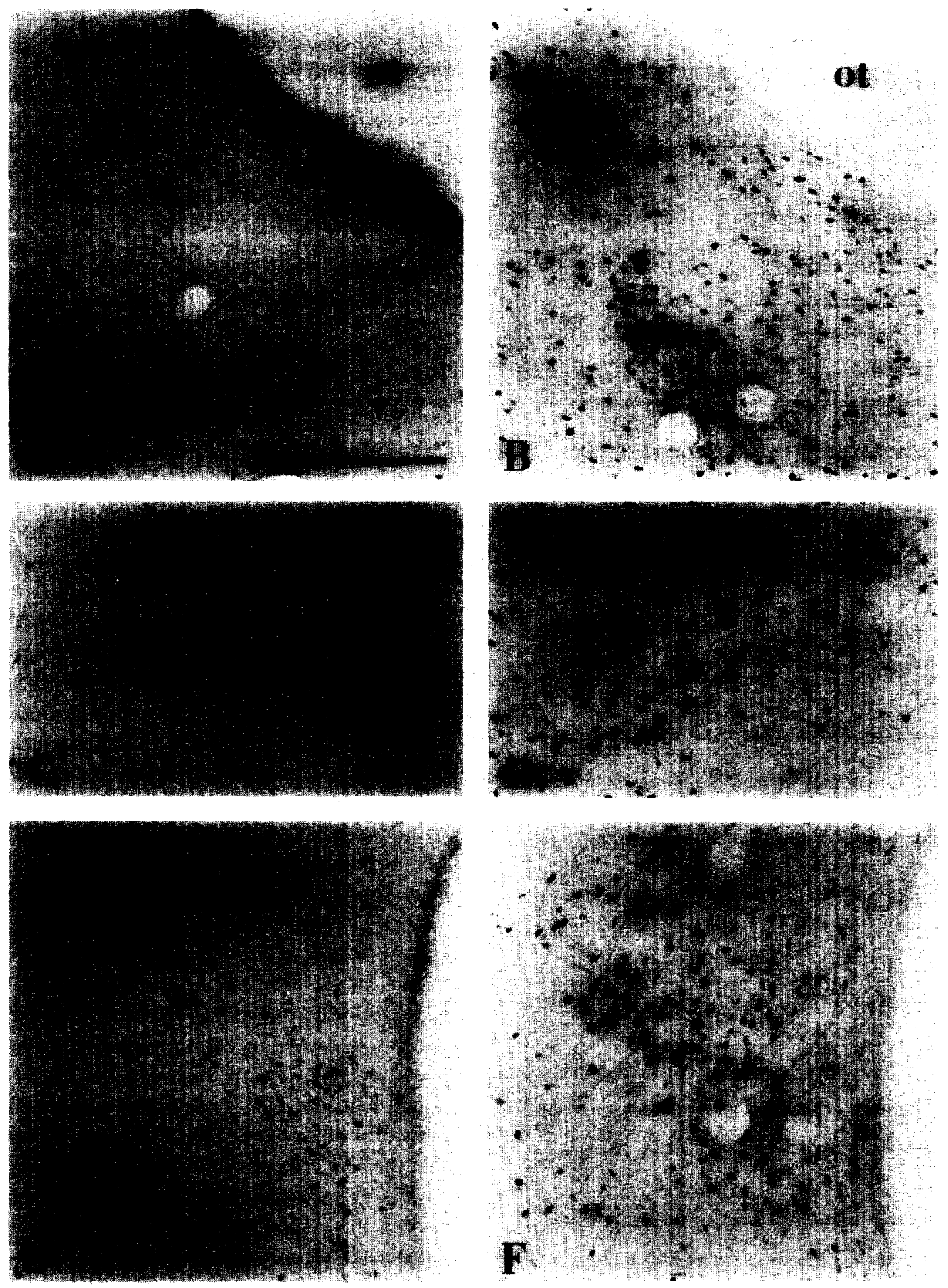

Fig. 2. Photomicrographs of Fos-ir neurons in non-mated (left column) and mated (right column) hamsters: (A,B) caudal posterodorsal subdivision of Me (cMePD), (C,D) magnocellular medial preoptic nucleus (MPNmag), and (E,F) paraventricular nucleus of the hypothalamus (PVN). $\operatorname{Bar}=200 \mu \mathrm{m}$.

and penile musculature during mating behavior. In the rat, neurons located largely within the parvocellular subdivisions of the PVN project to several sites within the brain stem and spinal cord that regulate the autonomic nervous system [23] and to motoneurons within the lumbar spinal cord that innervate the penile musculature $[15$,
22]. In addition, preliminary data suggest that sexual behavior induces Fos expression in the rat PVN and that a subset of these neurons contain oxytocin [7]. The connections and neurochemical content of Fos-ir neurons in the hamster PVN await further study.

Although mating was the basis for Fos induction in 
A

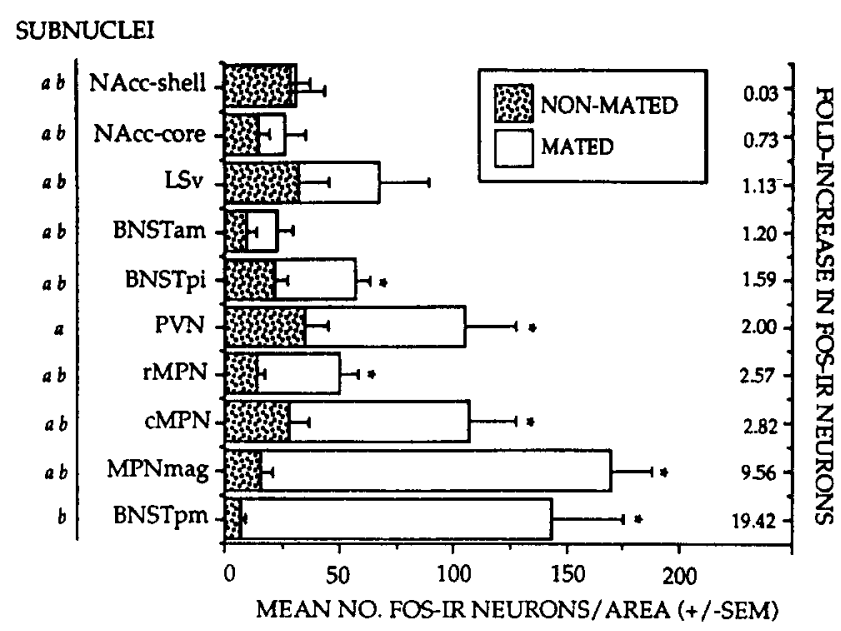

B
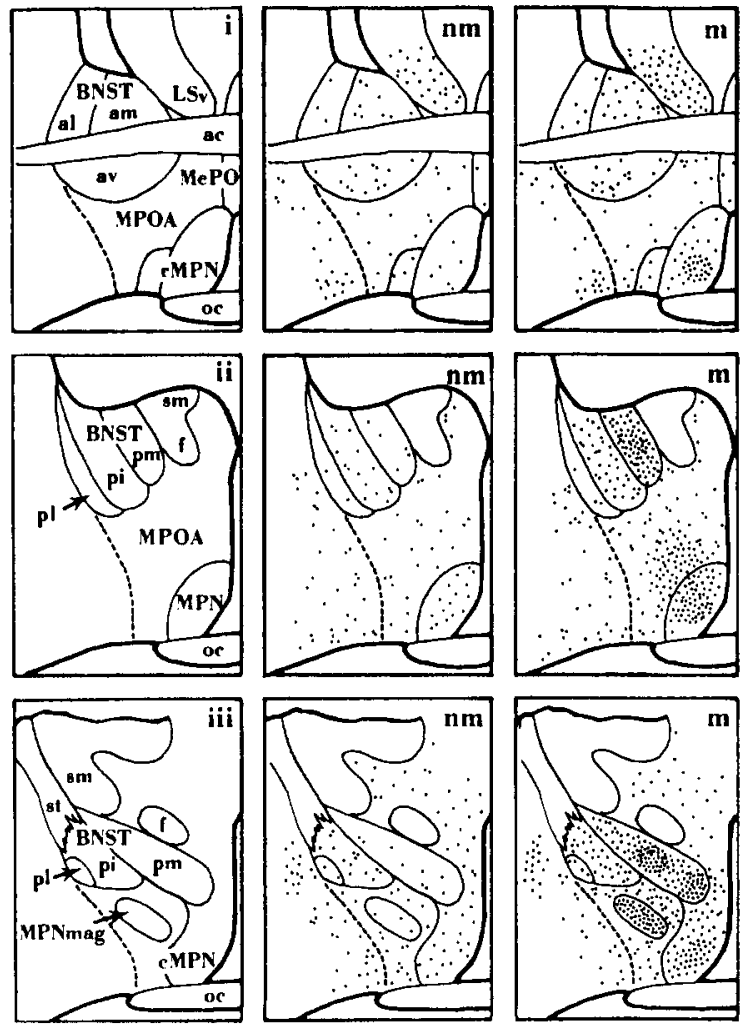

$\leftarrow$

Fig. 3. A: mean number of Fos-ir neurons per subnucleus in the BNST and MPOA of non-mated and mated groups. The fold-increase in Fosir neurons indicates the mating-induced increase in Fos-ir neurons above basal levels. An asterisk identifies those subnuclei that contained significantly more Fos-ir neurons following mating. The letters to the left of each subnuclei identify differences between subnuclei in nonmated animals. Subnuclei that share a common letter do not differ significantly. B: 9-panel schematic illustrates the distribution of Fos-ir neurons between non-mated $(\mathrm{nm})$ and mated $(\mathrm{m})$ groups at three different anatomical levels through the BNST and MPOA. ac, anterior commissure; BNST, bed nucleus of the stria terminalis; am, anteromedial BNST; al, anterolateral BNST; av, anteroventral BNST; pi, posterointermediate BNST; pl, posterolateral BNST; pm, posteromedial BNST: f, fornix; LSv, ventral lateral septum: MePO, median preoptic nucleus: MPN, medial preoptic nucleus; rMPN, rostral part of MPN; cMPN, caudal part of MPN; MPNmag, magnocellular MPN; MPOA, medial preoptic area; NAcc, nucleus accumbens (shell, core); PVN, paraventricular nucleus of the hypothalamus: sm. stria medullaris; st, stria terminalis

The significance of Fos expression within individual neurons along the chemosensory pathways is also unknown. Fos protein forms part of the transacting factor AP-1 that controls the transcription of target genes [5, 18]. The induction of Fos protein may stimulate gene transcription, for example, to replenish stores of neurotransmitters expended during behavior, or to mediate changes in the processing of chemosensory and hormonal signals seen after sexual experience [12, 14]. Determining the transcriptional events that result from c-fos induction in the chemosensory pathways of sexuallynaive males will tell us much about the molecular basis of this complex behavior.

The authors wish to thank Lorita Dudus and Kaye Brabec for technical assistance. This work was supported by NIH NS20629 to S.W.N. and the Morphology Core Center Grant of the Reproductive Sciences Program HD19258.

1 Baum, M.J. and Everitt, B.J.. Immediate early genes induced following sexual behaviour in male rats: differential expression of $\mathrm{c}-\mathrm{fos}$ in limbic structures, Soc. Neurosci. Abstr., 17 (1991) 1059.

2 Cattaneo. E. and Maggi, A.. c-fos induction by estrogen in specific rat brain areas, Eur. J. Pharmacol. 188 (1990) 153-159.

3 Doherty. P.C. and Sheridan, P.J., Uptake and retention of androgen in neurons of the brain of the golden hamster. Brain Res.. 219 (1981) 327334 .

4 Fiber, J.M. and Swann, J.M., Female hamster vaginal secretion stimulates $\mathrm{c}$-fos expression in the vomeronasal and olfactory mating behavior pathways in the male golden hamster, Soc. Neurosci. Abstr. 17 (1991) 1060.

5 Franza, B.R., Jr., Rauscher, F.J. III, Josephs. S.F. and Curran, T., The Fos complex and Fos-related antigens recognize sequence ele- 
ments that contain AP-1 binding sites, Science, 239 (1988) 1150 1153.

6 Hunt, S.P., Pini, A. and Evan, G., Induction of c-fos-like protein in spinal cord neurons following sensory stimulation. Nature, 328 (1987) 632-634

7 Insel, T.R. and Witt, D.M., Activation of c-fos-like protein in PVN oxytocin cells during male sexual behavior, Soc. Neurosci. Abstr. 17 (1991) 1415 .

8 Insel, T.R., Regional induction of c-fos-like protein in rat brain after estradiol administration, Endocrinology, 126 (1990) 1849 1853.

9 Lehman, M.N. and Winans, S.S., Vomeronasal and olfactory pathways to the amygdala controlling male hamster sexual behavior: autoradiographic and behavioral analyses, Brain Res., 240 (1982) $27-41$

10 Lehman, M.N., Powers, J.B. and Winans, S.S., Medial nucleus of the amygdala mediates chemosensory control of male hamster sexual behavior, Science, 210 (1980) 557-560.

11 Lehman, M.N., Powers, J.B. and Winans, S.S., Stria terminalis lesions alter the temporal pattern of copulatory behavior in the male golden hamster, Behav. Brain Res., 8 (1983) $109 \quad 128$

12 Lisk, R.D. and Heimann, J., The effects of sexual experience and frequency of testing on retention of copulatory behavior following castration in the male hamster, Behav. Neural Biol., 28 (1980) 156 . 171.

13 Macrides, F., Bartke, A., Fernandez, F. and D'Angelo, W., Effects of exposure to vaginal odor and receptive females on plasma testosterone in the male hamster, Neuroendocrinology, 15 (1974) 355 364.

14 Meredith, M., Vomeronasal organ removal before sexual experience impairs male hamster mating behavior, Physiol. Behav., 36 (1986) 737-743

15 Monaghan, E.P. and Breedlove, S.M., Brain sites projecting to the spinal nucleus of the bulbocavernosus, J. Comp. Neurol., 307 (1991) $370-374$

16 Morgan, J.I., Cohen, D.R., Hempstead, J.L. and Curran, T., Mapping patterns of $\mathrm{c}$-fos expression in the central nervous system after seizure, Science, 237 (1987) 192 197.
17 Powers, J.B., Newman, S.W. and Bergondy, M.L., MPOA and BNST lesions in male Syrian hamsters: differential effects on copulatory and chemoinvestigatory behaviors, Behav. Brain Res., 23 (1987) 181-195.

18 Rauscher, F.J. III, Sambucetti, L.C., Curran, T., Distel, R.J. and Spiegelman, B.M., Common DNA binding site for Fos protein complexes and transcription factor AP-1, Cell, 52 (1988) $471-480$.

19 Robertson, G.S., Pfaus, J.G., Atkinson, L.J., Matsumura, H., Phillips, A.G. and Fibiger, H.C., Sexual behavior increases c-fos expression in the forebrain of the male rat, Brain Res., 564 (1991) 352-357

20 Sachs, B.D. and Meisel, R.L., The physiology of male sexual behavior. In E. Knobil and J. Neill (Eds.), The Physiology of Reproduction, Raven, New York, 1988, pp. 1403-1412.

21 Sagar, S.M., Sharp, F.R and Curran, T., Expression of c-tos protein in brain: metabolic mapping at the cellular level. Science, 240 (1988) 1328-1331.

22 Shen, P.. Arnold, A.P. and Micevych, P.E., Supraspinal projections to the ventromedial lumbar spinal cord in adult male rats, J. Comp. Neurol., 300 (1990) 263-272

23 Swanson, L.S. and Kuypers, H.G.J.M., The paraventricular nucleus of the hypothalamus: cytoarchitectonic subdivisions and the organization of projections to the pituitary, dorsal vagal complex and spinal cord as demonstrated by retrograde fuorescence doublelabeling methods, J. Comp. Neurol., 194 (1980) 555 570 .

24 van Straaten, F. Muller, R., Curran, R., Van Beveren, C. and Verma, I.M., Nucleotide sequence of a human $c$-onc gene: deduced amino acid sequence of human c-fos protein, Proc. Natl. Acad. Sci. USA, 80 (1983) $3183 \quad 3187$.

25 Weisz, A. and Rosales, R.. Identification of an estrogen response element upstream of the human $c$-fos gene that binds the estrogen receptor and the AP-1 transcription factor, Nucleic Acids Res., 18 (1990) 5097-5106

26 Wood, R.I., Brabec, R.K., Swann, J.M. and Newman, S.W., Androgen and estrogen concentrating neurons in chemosensory pathways of the male Syrian hamster brain. Brain Res., in press. 